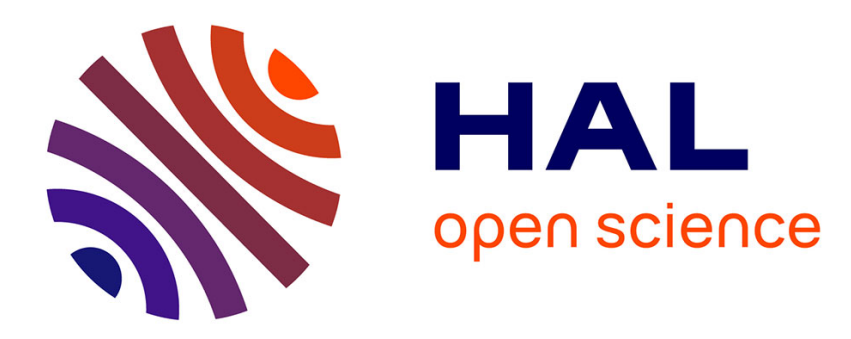

\title{
Le 6 juin 1892 : visite du Président de la république à l'Ecole nationale des eaux et forêts.
}

\author{
Jean Pardé
}

\section{To cite this version:}

Jean Pardé. Le 6 juin 1892 : visite du Président de la république à l'Ecole nationale des eaux et forêts.. Revue forestière française, 1998, 50 (5), pp.479-481. 10.4267/2042/5562 . hal-03443619

\author{
HAL Id: hal-03443619 \\ https://hal.science/hal-03443619
}

Submitted on 23 Nov 2021

HAL is a multi-disciplinary open access archive for the deposit and dissemination of scientific research documents, whether they are published or not. The documents may come from teaching and research institutions in France or abroad, or from public or private research centers.
L'archive ouverte pluridisciplinaire HAL, est destinée au dépôt et à la diffusion de documents scientifiques de niveau recherche, publiés ou non, émanant des établissements d'enseignement et de recherche français ou étrangers, des laboratoires publics ou privés. 


\section{LE 6 JUIN 1892 : VISITE DU PRÉSIDENT DE LA RÉPUBLIQUE À L'ÉCOLE NATIONALE DES EAUX ET FORÊTS}

Le lundi 6 juin 1892, à l'occasion d'un voyage officiel qu'il faisait en Lorraine, le Président de la République, Sadi Carnot, fit visite à l'École nationale des Eaux et Forêts.

Une visite dont on gardera volontiers souvenir, puisqu'elle fut - et demeure - la seule qu'un Président de la République - III ${ }^{\mathrm{e}}$ IV ${ }^{\mathrm{e}}$ ou Ve - fit à cette école.

Voici, pour en témoigner :

- une photographie de cet événement, représentant les personnalités dans la cour d'honneur de l'École (p. 480);

- le compte rendu qui en fut publié dans la Revue des Eaux et Forêts en 1892, pages 319-321.

Ce texte transporte - agréablement - le lecteur d'aujourd'hui dans une ambiance et une atmosphère bien éloignées des années qui terminent le $\mathrm{XX} \mathrm{X}^{\mathrm{e}}$ siècle.

Le Président Sadi Carnot, aux mérites reconnus par tous, était petit-fils du grand Lazare Carnot, "l'organisateur de la Victoire" de la Révolution française.

Il fut assassiné à Lyon, le 24 juin 1894, par un anarchiste italien, Santo Caserio.

Il est inhumé au Panthéon à côté de son illustre aïeul.

"Le lundi 6 juin, l'École forestière a reçu la visite de M. le Président de la République qui se trouvait à Nancy pour les fêtes du XVIII e congrès des sociétés de gymnastique.

Cette visite n'était pas d'abord comprise dans le programme, et malgré une démarche que M. Puton, directeur, s'était empressé de faire à Paris, les forestiers n'avaient reçu qu'une réponse décourageante. L'École tenait cependant à manifester quand même ses sentiments de patriotique dévouement, et un arc de triomple préparé par ses soins s'élevait rue de la Constitution (1), à défaut de la rue Girardet qui ne devait pas être parcourue par le cortège officiel.

Enfin, grâce à l'intervention des députés et sénateurs du département, un heureux changement put être obtenu et, le vendredi soir, une dépêche de Paris annonçait que M. Carnot consacrerait à l'École quelques instants. II restait bien peu de temps pour préparer au chef de l'État une réception digne de lui : néanmoins tous se mirent à l'œuvre, professeurs et élèves, et le résultat fut aussi satisfaisant qu'on pouvait l'espérer avec les faibles moyens laissés à la disposition du personnel forestier.

(1) Actuelle rue Maurice-Barrès. 
II y a...

À 10 heures du matin, l'arc de triomphe était prêt, lorsque le Président et sa suite traversèrent la rue de la Constitution pour se rendre au pont d'Essey et à Malzéville. C'était une construction originale et pittoresque, rapidement élevée sous la direction de MM. les professeurs Thiéry et Peticollot : deux énormes troncs de sapin, brisés à mi-hauteur, un volis arrêté transversalement et formant portique, le tout encadré de mousses, de drapeaux, d'inscriptions et de panneaux garnis de têtes d'animaux sauvages et des principaux attributs forestiers. Tout autour de ce monument, trente gardes forestiers en armes faisaient la haie ; l'un d'eux, le brigadier Fissabre, de Faulx (Meurthe-et-Moselle), venait de recevoir la médaille militaire, qui lui avait été remise au titre militaire par le Président de la République.

À 2 heures, M. Carnot arrivait à l'École forestière. La rue Girardet se trouvait ornée depuis la veille de mâts et de guirlandes; les bâtiments de l'École étaient pavoisés à toutes les fenêtres. Les préposés, commandés par M. le garde général Crettiez, ont rendu les honneurs militaires au Président dans la cour d'entrée, où étaient rassemblés les professeurs et les agents de la conservation de Nancy. M. Puton, directeur, accompagné de M. Lamblé, conservateur des forêts, et de M. Boppe, sous-directeur, attendait $M$. Carnot à la porte extérieure. Après avoir présenté les professeurs et les agents, M. Puton a conduit M. le Président de la République dans la grande Cour, où un velum orné

École nationale des Eaux et Forêts Réception du Président Sadi Carnot Collection ENGREF - Nancy

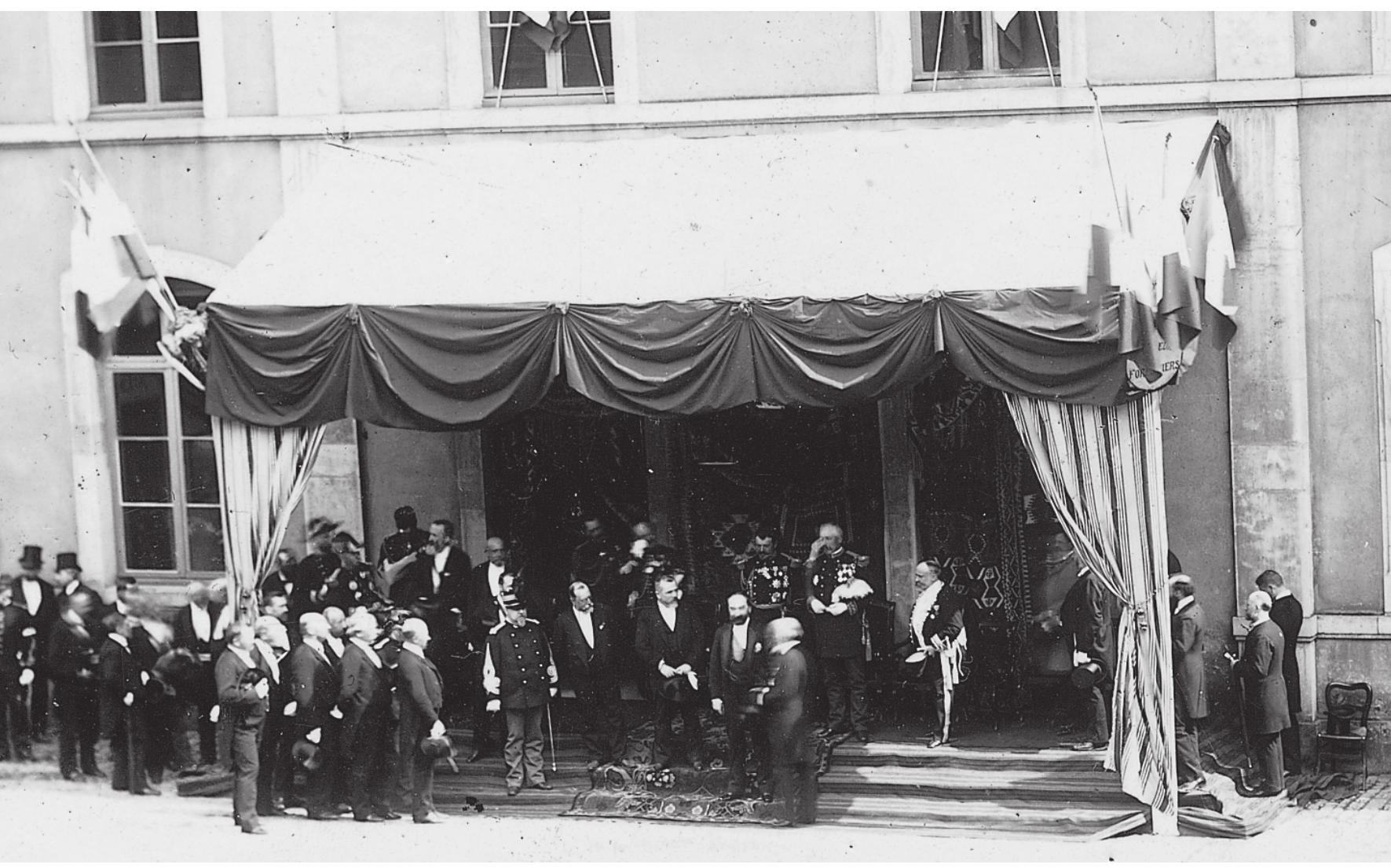


II y a...

\section{Arc de triomphe dressé par l'École en l'honneur du Président de la République Sadi Carnot}

Collection ENGREF - Nancy

de tapisseries avait été préparé ; des faisceaux de drapeaux, des cordons de feuillages et de jeunes sapins envoyés des forêts des Vosges faisaient face au perron et complétaient l'ornementation de cette partie des bâtiments.

Dans la suite de M. Carnot, on remarquait : M. Loubet, ministre de l'Intérieur et président du Conseil, M. Léon Bourgeois, ministre de I'Instruction publique, les généraux Jamont et Brugère, M. Volland, sénateur, MM. Cordier et Papelier, députés de Meurthe-et-Moselle, M. le Préfet et M. le Maire de Nancy, ainsi que de nombreuses notabilités de la ville et du département.

M. Puton a prononcé alors l'allocution suivante:

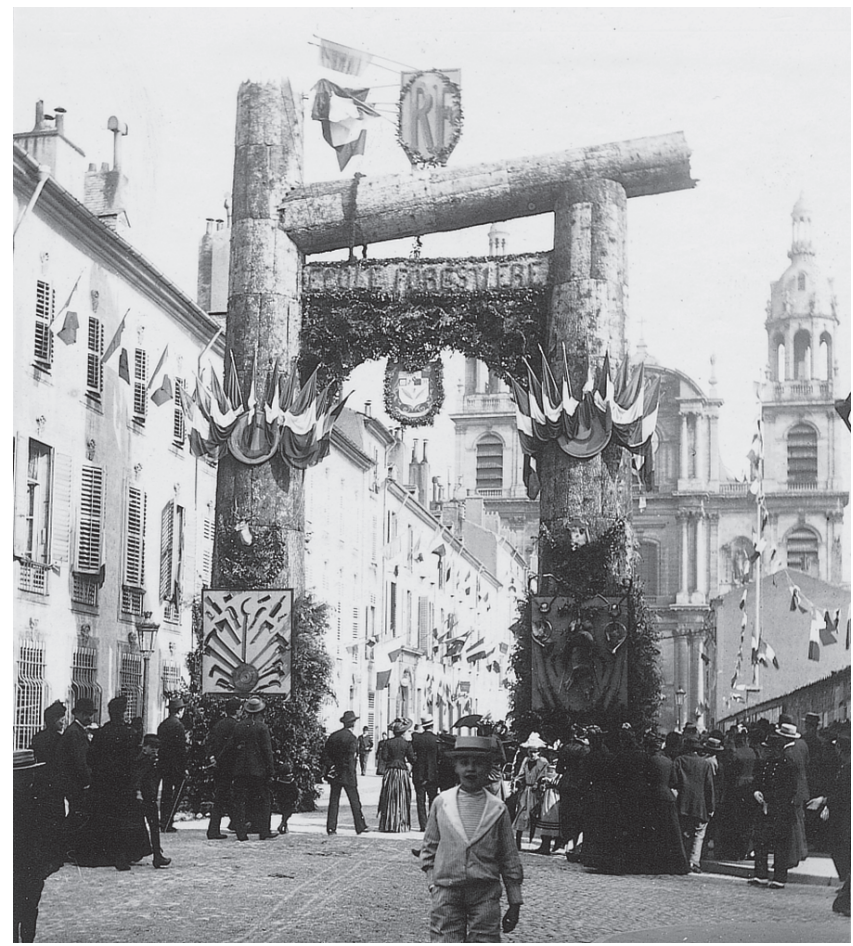

"M. le Président de la République,

Je ne saurais trop vous dire combien l'École forestière, ses maîtres et ses élèves, sont heureux de la visite que vous voulez bien leur faire et de la marque d'attention que vous donnez ainsi au corps forestier tout entier.

Nous savons tous ici que la République est le seul gouvernement qui n'ait pas vendu un seul hectare des forêts de l'État, qu'elle a acheté au contraire plus de soixante-dix mille hectares de terrain à reboiser en montagne, comme ressource de l'avenir.

Nous savons tous ici qu'elle est le premier gouvernement qui ait considéré le producteur de bois comme aussi digne de protection douanière que le producteur industriel, établissant ainsi l'égalité entre tous ceux qui fournissent les éléments du travail national.

À ces services s'ajoute l'intérêt que vous témoignez en ce jour à l'enseignement supérieur forestier. Je vous en exprime toute la reconnaissance de l'École ".

M. Carnot a répondu en s'informant avec sollicitude des conditions de l'enseignement et des résultats de la réforme qui recrute le personnel forestier dans l'Institut agronomique.

Les élèves, commandés par M. le lieutenant-colonel Montignault ont ensuite défilé, le sabre au poing. Après ce défilé, $M$. le Président a visité la grande galerie des collections de l'École, en passant par la salle des Pas-Perdus, ornée d'une manière très artistique par les élèves eux-mêmes. Il a enfin été reconduit à sa voiture, les élèves s'étant massés sur deux rangs pour le saluer à son départ.

Cette visite a été, en somme, un événement heureux pour l'École forestière. $M$. Loubet, président du Conseil des ministres, a bien voulu manifester la satisfaction qu'il avait ressentie pour l'excellente tenue des élèves, en assurant que le souvenir de cette belle réception ne serait pas oublié ». 